\section{Electromagnetic jet propulsion: non-Iorentzian forces on currents?}

Two instances have been reported by Graneau ${ }^{1}$ of an electromagnetic force on a current element apparently acting in the direction of current flow, in contrast to the generally accepted Lorentz force which always acts at right angles to the current, and he believes that these observations support instead Ampère's older formula for the force acting between two current elements.

In the first place, however, both observations can be understood in terms of Lorentz forces, for in the examples given, the current flow lines diverge from a point of constriction, so that the locally generated magnetic field exerting a force normal to the current produces a component of force parallel to the axis of symmetry of the diverging current, directed away from the apex. A quantitative check is possible in the case of the "copper submarine', the long copper rod (radius $a=1.5 \mathrm{~mm}$ ), blunt at one end but tapered to a point at the other, which submerged itself when placed in a trough of mercury (0.25 sq. inch cross-section) carrying a 400 A current $(I)$, and travelled along the trough at a speed of $15 \mathrm{~cm} \mathrm{~s}^{-1}$, always with the pointed end trailing.

Because of the high conductivity of copper, much of the current will be funnelled into the copper not far from the point, from where the lines of current flow will spread apart somewhat on moving towards the wider part of the copper, and in this cone section the Lorentz force has a component parallel to the axis, the magnetic field lines being circles around the axis. (After leaving the blunt end, the current will again diverge, exerting a propulsive force on the mercury.) Taking the resistance per unit length of the mercury to be $k(\simeq 2.67)$ times that of the copper at its full width, and assuming that in any plane normal to the central axis the current density is nearly uniform in each medium and in proportion to conductivity, the Lorentz forces can be integrated over the whole of the cone section of the copper, to give the total longitudinal force $\mu_{0} I^{2} F / 4 \pi$ (SI units), where $F=\frac{1}{2}\left\{\ln (1+k)-(k / 1+k)-\frac{1}{2}(k / 1+k)^{2}\right\}$, directed away from the apex. The motion is resisted essentially by the inertial force $\approx \rho v^{2} \pi a^{2}$ (neglecting any motion of the fluid), $\rho$ being the density of mercury. This gives a velocity $v=0.16 \mathrm{~m} \mathrm{~s}^{-1}$, in good agreement with observation.

In the other observation cited, where mercury flowed away from the end of a stationary wire carrying a current, the current would fan out into the mercury and so exert a force near the point of divergence, directed away from the wire.

Second, one cannot distinguish by experiment between the two different laws for the mutual forces acting on two current elements, for one can observe only the total force acting on a current element, and not which element is the source of this force. It was established long ago ${ }^{2,3}$ that both laws yield identical results for the force on a current element exerted by a current which flows in a complete circuit.

\section{A. M. Hillas}

Department of Physics,

University of Leeds,

Leeds LS2 9JT, UK

1. Graneau, P. Nature 295, 311-312 (1982)

2. Maxwell, J. C. Treatise on Electricity and Magnetism Vol. 2, 160-161, 290 (Clarendon, Oxford, 1873).

3. O'Rahilly, A. Electromagnetics, 106-108 (Longmans, Green, London, 1938).

GRANEAU REPLIES-Hillas believes that the motive force in the two experiments is being provided by certain components of the Lorentz force on the hairpin bend and the conical part of the submarine. These components have no corresponding equal and opposite reaction forces in the liquid mercury or any other material medium. They may be termed self-forces, giving rise to self-propulsion. In the strict sense of special relativity theory, they are endowed with reaction forces in the field (vacuum) which accelerate matterless electromagnetic mass. Forces on vacuum may not be counted towards the force balance required by Newton's third law. On this issue Vannevar Bush ${ }^{2}$, Cleveland ${ }^{3}$, O'Rahilly ${ }^{4}$, Whittaker ${ }^{5}$ and Robertson ${ }^{6}$ aligned themselves with the newtonian dynamics. Their ranks must have swollen considerably since space travel has become a reality, for nobody tries to exploit vacuum reaction forces in pursuit of novel methods of space propulsion.

Hillas' references to Maxwell and O'Rahilly concern the analysis of rectangular circuits and not arbitrarily shaped, asymmetrical circuits. It is an accident of symmetry that the self-forces on the two parts of a rectangular circuit are equal and opposite and of the same magnitude as the Ampère reaction forces. For an analysis of unsymmetrical circuits of any shape one has to go back to the writings of Ampère ${ }^{1}$.

Hillas is correct in maintaining that the Ampère and the Lorentz force on an isolated current-element are the same when produced by the current in a separate closed circuit. But this is not true when the isolated current-element is incorporated in the closed circuit. Instead of relying on Ampère's long analysis, this can today be verified by finite current-element analysis with computers. It will then be found that, around an arbitrarily shaped circuit, the Ampère force has everywhere a tangential component in addition to the transverse component, the latter being equivalent to the Lorentz force. The tangential or longitudinal Ampère forces are consistent with the generation of tension in straight wires and jet-reactions at liquid-solid conductor interfaces.

The Lorentz force was tailored to the electron moving through vacuum. No compelling argument seems to have been made for the moving free electron to be the same entity as an amperian metallic current-element. Perhaps there is room for two force laws?

\section{P. GRaneau}

Francis Bitter National Magnet Laboratory of Technology, Massachusetts Institute, Cambridge, Massachusetts 02139, USA

1. Ampere, A. M. Memoires sur l'Electrodynamic Vol. 1,25 (Gauthier-Villars, Paris, 1885).

2. Bush, V. J. Math. Phys. 5, 129 (1926).

3. Cleveland, F. F. Phil. Mag. 25, 416 (1936).

4. O'Rahilly, A. Electromagnetic Theory Vol. 1, 102 (Dover, New York, 1965)

5. Whittaker, E. History of the Theories of Aether and Elec tricity Vol. 1, 84 (Nelson, London, 1951).

6. Robertson, I. A. Phil. Mag. 36, 32 (1945).

\section{The significance of transient haematopoietic spleen colonies}

RECENTLY new and interesting observations have been reported by Magli et al. ${ }^{1}$, which led them to question the validity of the widely used 'spleen colony' assay for multipotential stem cells in blood forming tissues. Their observation was that the colonies of cells in the spleen 7 days after transplantation of bone marrow into irradiated mice were only transient, and so cannot be considered as measuring cells with extensive proliferative capacity. Their conclusion has been reached through insufficient attention being given to existing experimental data and failure to distinguish carefully between the capacity of the cells (their nature) and the particular demands made upon them (their nurture).

Many years ago Siminovitch et $a l^{2}$ pointed out that if differentiation was a stochastic event there should be a wide variation in the number of stem cells in colonies derived from single cells and he reported measurements that fitted the expected highly skewed distribution. Later Vogel et al. $^{3}$ estimated the 'extinction' coefficient, that is, the proportion of stem cells reaching the spleen which differentiate before being able to establish a colony. Therefore some stem cells will fail to produce any colonies, while others can be expected to run out of stem cells later, thus producing transient colonies, caused by the pressure of differentiation in the spleen, without the cells necessarily lacking self-renewal capacity.

A further aspect concerns the competing demands for stem cells to maintain both their own cell numbers and provide cells for differentiation. It is known that the population of cells measured by the 'spleen colony' assay and scored at 7 days is heterogeneous, comprising cells ranging from those with low proliferation rate and high capacity for self-renewal to cells with 